\title{
Management of Non-Prosthetic Peri-Implant Fracture in a Case of Broken Intramedullary Locking Nail - A Rare Case Report and Review of Literature
}

\author{
Amit supe ${ }^{1}$, Jayesh Anant Mhatre ${ }^{2 *}$, Nihar Modi ${ }^{3}$ \\ Department of Orthopaedics, Grant Government Medical College, Mumbai, Maharashtra, India
}

*Corresponding Author: Jayesh Mhatre, Department of Orthopaedics, Grant Government Medical College, Mumbai, Maharashtra, India..

Received Date: December 14, 2021; Accepted Date: December 30, 2021; Published Date: January 12, 2022

Citation: Amit supe, Jayesh Anant Mhatre, Nihar modi (2022). Management of Non-Prosthetic Peri-Implant Fracture in a Case of Broken Intramedullary Locking Nail - A Rare Case Report and Review of Literature. J. Clinical Orthopedics and Trauma Care, 4(1); DOI:10.31579/26940248/024

Copyright: (C) 2022 Jayesh Mhatre, This is an open access article distributed under the Creative Commons Attribution License, which permits unrestricted use, distribution, and reproduction in any medium, provided the original work is properly cited.

\begin{abstract}
A non-prosthetic peri-implant fracture (NPPIF) can be defined as a fracture in a bone with an existing non-prosthetic implant such as an extramedullary plate and screws or an intramedullary nail, NPPIFs are mostly reported together with peri-prosthetic fractures (PPFs) that occur around joint replacement implants, but they represent a separate clinical entity with different conceptual and practical considerations 1 . Our case is a 72-year male with a broken humerus intramedullary Nail in right upper limb who was managed with implant removal and bridge plating with locking plate. The favourable outcome in our case sheds a light in a grey region of non-prosthetic peri implant fractures of upper limbs where no definite management protocol is available. Our patient had very good outcome which was noted by 0/100 on dash scores and complete pain-free range of movement elbow and shoulder. Our case stands as a manifest for NPPIFs of humerus, which can be managed with similar protocol which was used in our case.
\end{abstract}

Keywords: broken humerus nail; bridge plating

\section{Introduction}

A non-prosthetic peri-implant fracture (NPPIF) can be defined as a fracture in a bone with an existing non-prosthetic implant such as an extramedullary plate and screws or an intramedullary nail. In our case the patient was operated with intramedullary humerus nail 20 years back, mostly are NPPIFs reported together with peri-prosthetic fractures (PPFs) that occur around joint replacement implants, but a separate clinical entity with different conceptual and practical considerations. PPFs (periprosthetic fractures) have been extensively studied and there are various classification systems and modalities for treatment in the literature. In contrast, the literature addressing NPPIFs is rare [1].

Epidemiology and other social parameters related to diaphyseal humeral fractures have not been extensively studied as those related to fractures occurring in other parts of the human skeleton, such as the proximal femur or the distal radius. Never- theless, the available bibliographical resources report that the general incidence of humeral shaft fractures remain in the area to $1 \%$ to $2 \%$ of all fractures occurring in the human body and $14 \%$ of all fractures of the humerus [2].

The first description of a diaphyseal humeral fracture goes back to ancient Egypt and has been recorded on the Edwin Smith Papyrus, the world's oldest surviving surgical text that was written in Egyptian hieratic script around the 17th century BC. The papyrus was discovered by Edwin Smith in the 1860s and it was recently decoded by James P. Allen of the Metropoli- tan Museum of Art in New York. The author of the papyrus described the fracture of the humerus and proposed conserva- tive treatment: "Thou shouldst place him prostrate on his back, with something folded between his two shoulder-blades; thou shouldst spread out his shoulders, and to stretch apart his upper arm until that break falls into its place. Thou shouldst make for him two splints of linen, (and) thou shouldst apply one of them to the inside of his arm, (and) the other of them to the underside of his arm. Thou shouldst bind it with cloth, (and) treat afterward with honey every day until he recovers." It is obvious that little has changed in the treatment of diaphyseal humeral fractures since ancient times, as humeral fractures heal within a short time ${ }^{3}$. During the treatment patients are mobile whereas shoulder and elbow joints compensate for some malalignment. However, patients in modern times demand faster union rates and earlier return to preinjury activities while preserving functionality and motion of nearby joints. Therefore, over the last few decades, we have witnessed significant advances in the field of surgical management of diaphyseal humeral fractures.

The most common reason for a humeral shaft fracture is a fall, followed by motor vehicle accident. Other causes that account for less than $10 \%$ of 


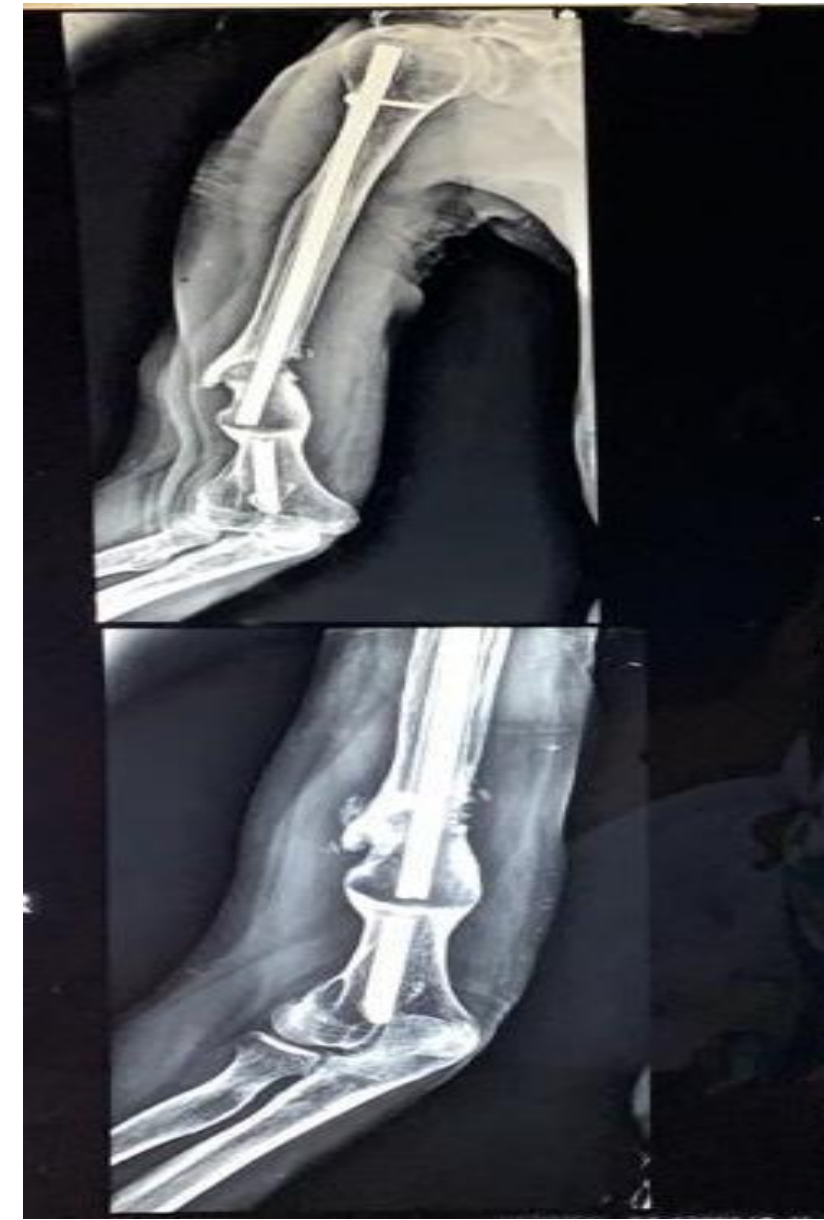

fall from a height, violence, and bone pathology. Pathologic and open fractures of the humeral shaft are uncommon (6\% to $8 \%$ and $2 \%$ to $5 \%$ of all diaphyseal humeral fractures, respectively) [4].

The commonest associated injury to a closed diaphyseal humeral fracture is the injury of the radial nerve (10\% to $12 \%$ of all closed humeral shaft fractures). The clinical manifestation is the inability to dorsiflex the wrist and digits while numbness occurs on the dorsoradial aspect of the hand and the dorsal aspect of the radial $3 \frac{1}{2}$ digits [3, 4].

Case Presentation: A 72-year-old gentleman, farmer by profession presented to our hospital with pain swelling and deformity over distal aspect of right upper arm of two days duration. patient was apparently well two days back, he sustained trauma to his right upper arm

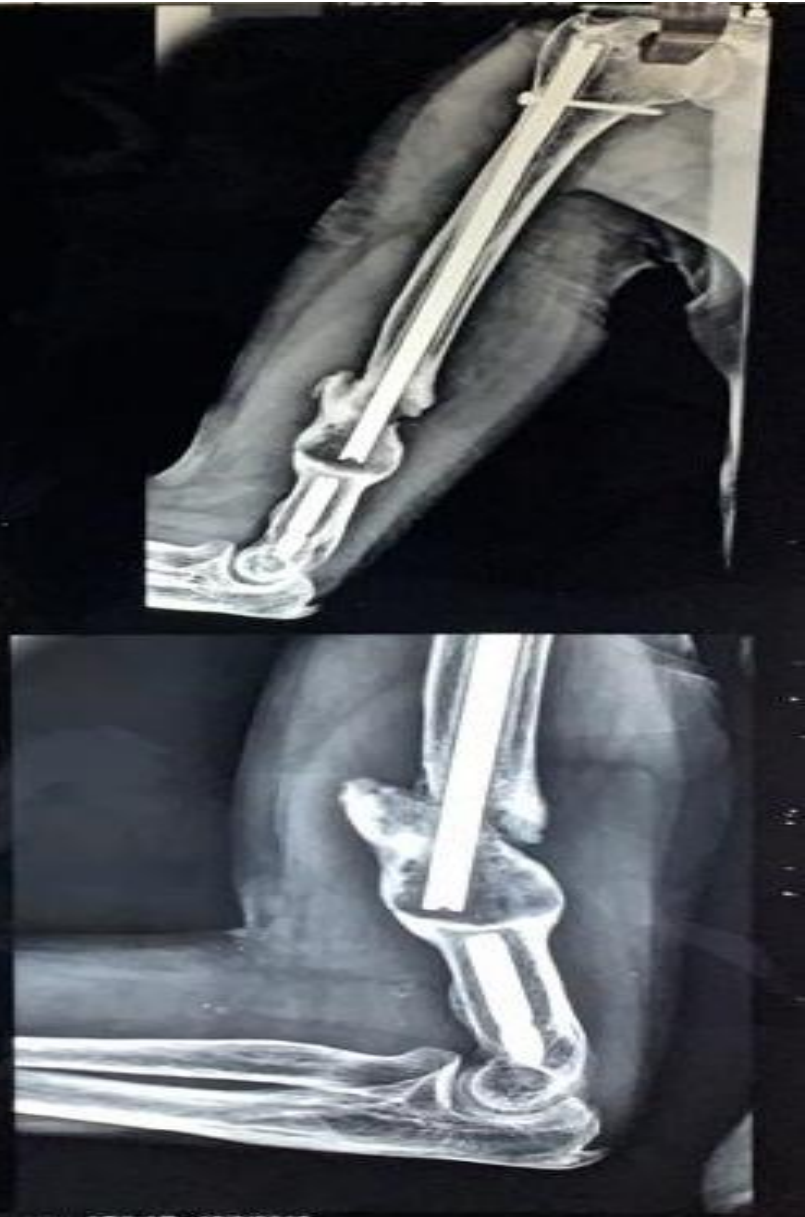

initial radiographs were done splintage was given and patient was referred to our hospital for further management. On clinical examination there was

swelling, tenderness and abnormal mobility along with crepitus over digital $1 / 3$ of right upper arm. He also gave a history of operation over same injured limb 20 years back, however no surgical details were available. Radiographs demonstrated a fracture of distal 1/3 of humerus with a broken intramedullary nail in-situ. Patient did not have distal neurovascular involvement or any other associated injuries and was relatively fit for surgery.He was posted for removal of the broken implant and fixation of fracture with open reduction internal fixation with the distal humerus pre-countered (LC-DCP) plate. 


\section{Surgery:}

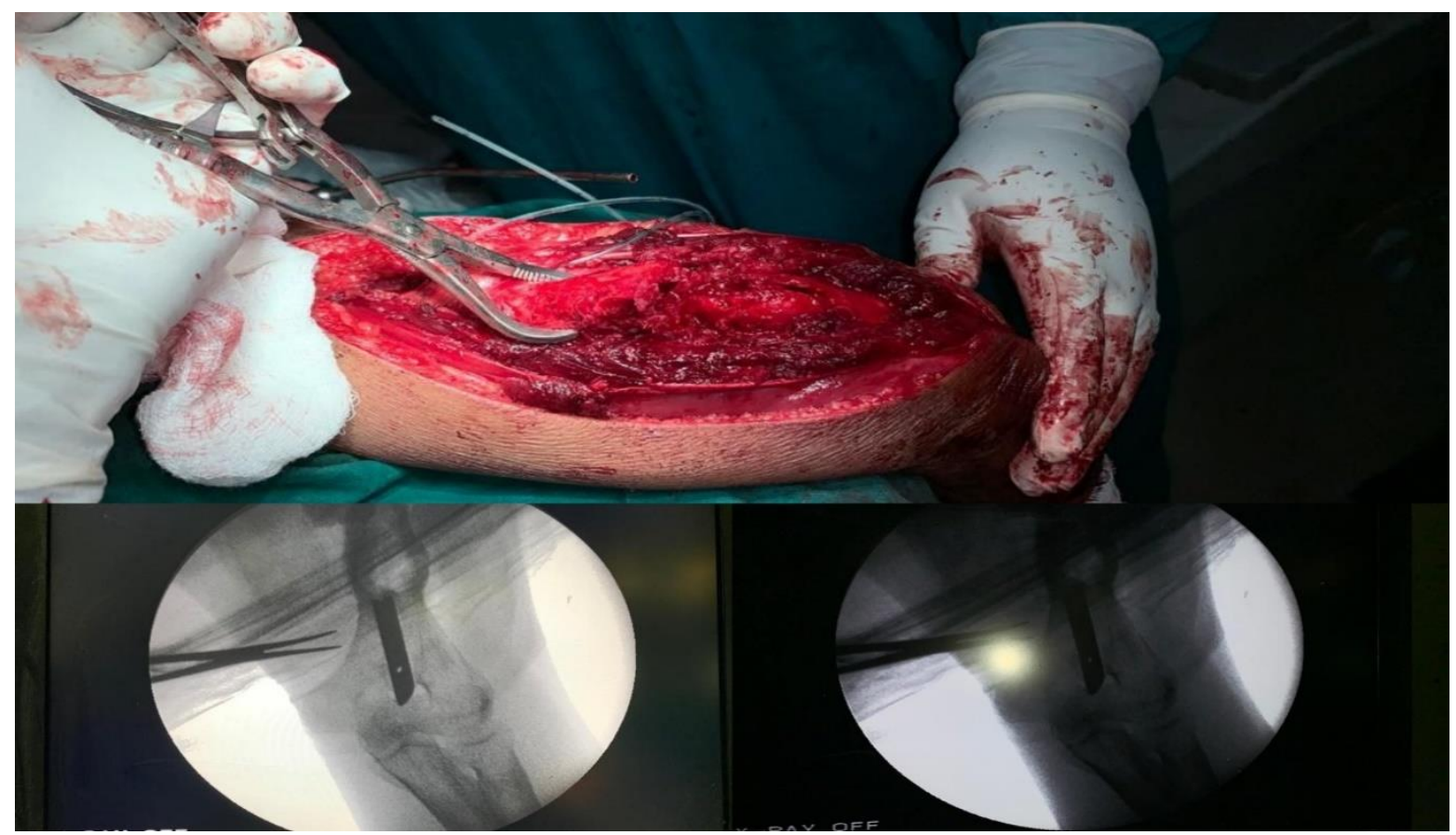

Figure 2:Intra-operative images.

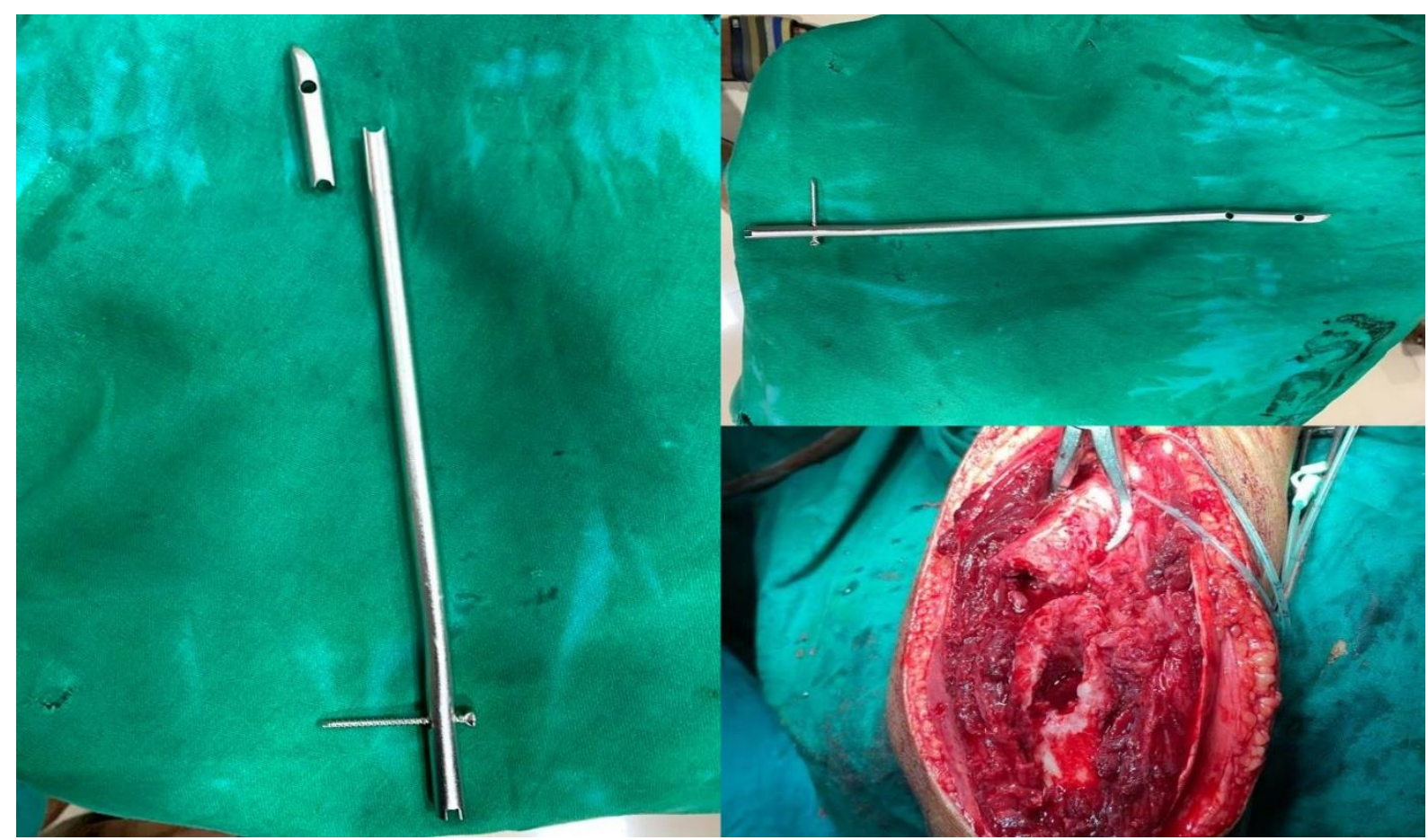

Figure 3: Images of the extracted nail.

Patient was and assessed for fitness of surgery, after all fitness tests and investigations, he was posted for open reduction internal fixation under supraclavicular and axillary block, in all aseptic conditions in lateral position with the arm abducted 90 degrees on a radiolucent support. Posterior approach is used to generally to expose the distal $2 / 3$ of humorous shaft, longitudinal incision in the midline of the posterior aspect of the arm from tip of the olecranon to about $7 \mathrm{~cm}$ distal to acromion was taken The dissection was initiated at the proximal end of the incision where the interval between the long and lateral head of the triceps was identified and developed by blunt dissection. The common tendon of the Biceps and triceps muscle was incised sharply in the midline, as it runs distally and inserts into the olecranon, then after retracting the lateral head of triceps laterally and the long head medially, at the proximal part of the incision, revealed the radial nerve and the profunda brachii artery as they run together in the spiral groove. Longitudinal midline dissection continued to reveal the distal humeral 
J. Clinical Orthopedics and Trauma Care

fracture site, fracture site was prepared with removal of excess fibrous tissue and then the distal tip of the broken nail was removed with a grasper as it did not have any locking screws, proximal screws were removed under image intensifier and then from proximal fracture fragment the proximal part of nail was removed, after removal of nail, reaming was done, as the fracture site was distal, the plate needed to be precontoured which is easiest with the use of special flexible templates the reduction was not achieved prior to plate application, so the plate was applied to the bone on one side of the fracture and the other side was aligned with its longitudinal axis. It was then provisionally secured to the bone with reduction clamps and fixed in this position with one screw. The next step done was to reduce the fracture by aligning the bone on the other side of the fracture with the plate. When the alignment was satisfactory, the unsecured end of the plate was clamped to the bone. Care was taken with the use of reduction clamps not to injure any of the neurovascular structures, which in the humerus may be close to the bone. Alignment was confirmed, both visually and with the image intensifier, and the remainder of the screws were inserted. The final reduction and the plate and screw lengths were confirmed with the image intensifier prior to wound closure. the fixation involved the exploration of a nerve that was nearby or crossing the plate (for instance, the radial nerve during the posterior approach), the exact relationship of the nerve to the plate was confirmed and recorded to avoid inadvertent placement of the plate on the nerve and reduce the risk of accidental nerve damage during plate removal at any time in the future. Closure was done in layers. Surgery was uneventful.

Post-operative protocol: for early mobilization of patient passive and active elbow and shoulder exercises were started found the 5th day of surgery. Suture removal was done on 12th day, wound was healthy there were no signs of infection. From second week of surgery patient was given functional humeral brace and full mobilization exercise was started. On follow up after 6 months of surgery patient had no lag in any range of movements of shoulder or elbow joint, his Distal Arm shoulder hand score was $0 / 100$.

\section{Discussion:}

NPPIFs are a sparsely reported, the orthopaedic literature has focused on fractures of the proximal femur with very less focus on upper extremity. In the earlier period the cephalo-medullary (CM) nails were introduced for fixation of peri-trochanteric fractures; CM nails had theoretical biomechanical advantages over extramedullary implants such as the sliding hip screw (SHS), unfortunately, these advantages were offset by a high rate of peri-implant fracture at the distal end of the nail. The literature regarding non femoral NPPIFs is extremely sparce [1].

Suboptimal surgical technique, weak implants, and poor bony purchase mainly because of osteoporosis have been recognized as the main reasons for Implant or Prosthesis failure. Intramedullary nailing has more implant specific complications. Dysfunction and pain at the shoulder has been regarded as the most common problem of antegrade intramedullary nailing of diaphyseal humeral fractures. However, many authors believe that nailing alone may not be responsible for this problem ${ }^{5}$. Nevertheless, iatrogenic injury to the rotator cuff could be avoided with the implementation of the retrograde technique or other approaches that spare the rotator cuff. Other complications that originate from the use of locking screws include neurologic damage and other soft tissue complications such as injury to arteries, muscles, and tendons. Injury to the long head of the biceps and the axillary nerve can be reduced by avoiding the proximal antero-posterior locking screw that many antegrade nails. Retrograde "fixed" nails reduce but do not abolish the incidence of injury to vulnerable soft tissues around the shoulder girdle [6]. Another problem of antegrade nailing is protrusion of the nail and impingement at the acromion. However, it is now recognized that antegrade nails must be embedded within the humeral head and protrusion and impingement should be regarded as technical error. Another under-reported problem of antegrade nailing is backing out of the proximal screws, because of the poor purchase within the can- cellous bone of the head, especially in osteoporotic patients. To overcome this problem some nails, provide either locking proximal screws or a polyethylene augmentation that covers the proximal screw holes and keeps the screws in situ. Sporadic complications of "fixed" nails include heat-induced seg- mental necrosis of the diaphysis and heterotopic ossification of the deltoid, both attributed to the reaming process and fracture at the tip of the nail $[6,7]$.

For diaphyseal humeral fractures the traditional 4.5-mm narrow dynamic compression plate (DCP) or the more recent limited contact dynamic compression plate (LC-DCP) is used. There should be a minimum of three to four holes in simple fractures an 8- to 10-hole plate should be sufficient, while in comminuted fractures it is recommended that the plate should span the area of comminution (bridging plate) requiring longer plates [7].

LC-DCP plates can also be used as they are easier to contour and offer the additional advantages of decreased stress shielding and preservation of blood supply of the periosteum because of the limited plate-bone contact.

One of the major issues that can affect treatment outcome with ORIF of a diaphyseal humeral fracture, before even fracture reduction and fixation, is the stripping and devitalization of bone fragments during the surgical approach. Another pitfall that could delay or inhibit bone healing is the inadequate reduction of the fracture. Although fracture reduction does not have to be anatomical in the humerus, lack of bone contact or a gap at the fracture site has a poor prognosis for union. Bone defects up to 2 to $3 \mathrm{~cm}$ should be dealt with shortening, as the arm can accommodate small discrepancies without functional consequences. Larger bone defects should be bridged with bone grafting, preferably autologous. The fixation technique must provide adequate stability with the plate being strong (4.5 $\mathrm{mm} \mathrm{DCP}$ ) and long enough to allow at least four holes and three to four screws in each main fragment although not every screw hole has to be filled.

Additional screws (e.g., lag screws) that enhance stability can be used. As a general principle, the chosen plate for the fixation should be positioned symmetrically to allow an equal number of screws on both sides of the fracture. Therefore, ORIF of distal humeral fractures could require screw placement in one or both condyles and even double plating to provide the necessary stability to the fracture $[8,9]$. Iatrogenic injury to neurovascular structures and most particularly to the radial nerve as it runs around the humerus must be avoided by identifying and protecting the neurovascular structures in most approaches. Apart from direct injury to the radial nerve during dissection, it should be noted that cerclage wiring, or drills and screws inserted from the opposite cortex can also injure the nerve [10].

\section{Conclusion:}

Our case presented a challenge, the patient was old, osteoporotic so healing of fracture was main concern which had to be addressed along with removal of the previous implant and provision of stability with new implant which could provide adequate healing pace. Most of the literature is focused on non-prosthetic peri-implant fractures of the hip or lower limb. The favourable outcome in our case sheds a light in a grey region of non-prosthetic peri implant fractures of upper limbs where no definite management protocol is available. Our patient had very good outcome which was noted by $0 / 100$ on dash scores and complete pain-free range of movement in elbow and shoulder. Our case stands as a manifest for NPPIFs of humerus, which can be managed with similar protocol which was used in our case 


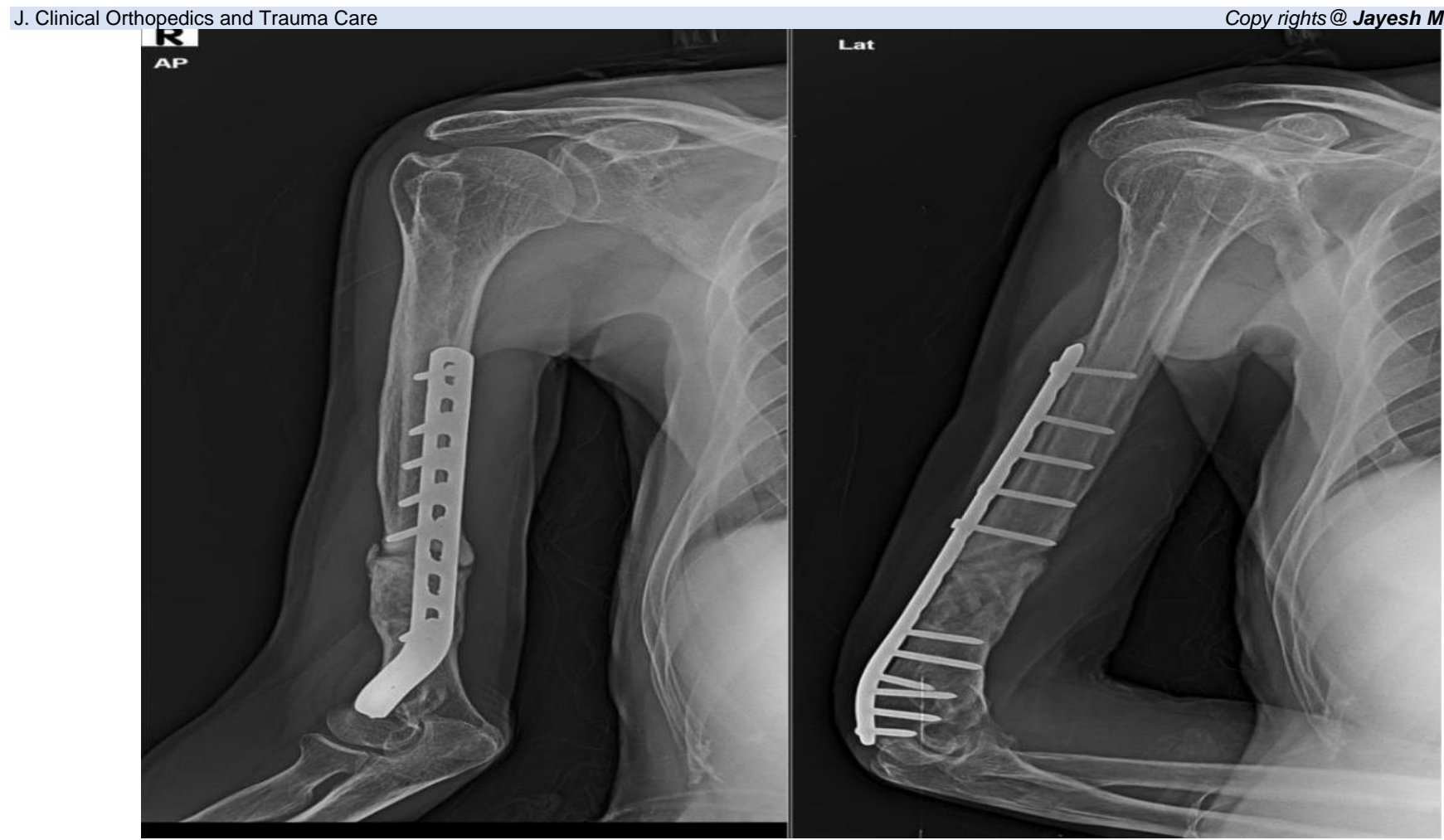

Figure 4: Radiograph at 6 month Follow-up period.

\section{Conflict of Interest: Nil}

\section{Funding: Nil}

Ethical approval: in Compliance with ethical standards.

Informed consent: well explained and taken.

\section{References:}

1. Chan, Lester \& Gardner, Antony \& Wong, Merng \& Chua, Kenon \& Kwek, Ernest Beng Kee. (2018). Non-prosthetic periimplant fractures: classification, management, and outcomes. Archives of Orthopaedic and Trauma Surgery. 138. 10.1007/s00402-018-2905-2901.

2. B. Livani and W. D. Belangero, "Bridging plate osteosynthesis of humeral shaft fractures," Injury, vol. 35, no. 6, pp. 587-595, 2004.

3. M. Walker, B. Palumbo, B. Badman, J. Brooks, J. Van Gelderen, and M. Mighell, "Humeral shaft fractures: a review," Journal of Shoulder and Elbow Surgery, vol. 20, no. 5, pp. 833844,2011

4. Chapman JR, Henley MB, Agel J, Benca PT. Randomized prospective study of humeral shaft fracture fixation: Intramedullary nails versus plates. Journal of Orthopaedic Trauma 2000;14(3):162 -166
5. Oh CW, Byun YS, Oh JK, Kim JJ, Jeon IH, Lee JH, Park KH. Plating of humeral shaft fractures: comparison of standard conventional plating versus minimally invasive plating. Orthopaedics\& Traumatology: Surgery \& Research 2012;98(1):54- 60

6. H. Seidel, "Humeral locking nail: a preliminary report," Orthopedics, vol. 12, no. 2, pp. 219-226, 1989

7. Changulani M, Jain UK, Keswani T. Comparison of the use of the humerus intramedullary nail and dynamic compression plate for the management of diaphyseal fractures of the humerus. A randomised controlled study. International orthopaedics 2007;31(3):391 - 395

8. P. Augat, J. Burger, S. Schorlemmer, T. Henke, M. Peraus, and L. Claes, "Shear movement at the fracture site delays healing in a diaphyseal fracture model," Journal of Orthopaedic Research, vol. 21, no. 6, pp. 1011-1017, 2003

9. D. C. Fitzpatrick, J. Doornink, S. M. Madey, and M. Bottlang, "Relative stability of conventional and locked plating fixation in a model of the osteoporotic femoral diaphysis," Clinical biomechanics, vol. 24, no. 2, pp. 203-209, 200

10. E. Fulkerson, K. A. Egol, E. N. Kubiak, F. Liporace, F. J. Kummer, and K. J. Koval, "Fixation of diaphyseal fractures with a segmental defect: a biomechanical comparison of locked and conventional plating techniques," The Journal of Trauma, vol. 60 , no. 4 , pp. $830-835,2006$ 
This work is licensed under Creative Commons Attribution 4.0 License

To Submit Your Article Click Here:

Submit Manuscript

DOI:10.31579/2694-0248/024
Ready to submit your research? Choose Auctores and benefit from:

$>$ fast, convenient online submission

$>$ rigorous peer review by experienced research in your field

$>$ rapid publication on acceptance

$>$ authors retain copyrights

$>$ unique DOI for all articles

$>$ immediate, unrestricted online access

At Auctores, research is always in progress.

Learn more https://auctoresonline.org/journals/journal-of-thoracic-disease-andcardiothoracic-surgery 